\title{
Efeito do óleo essencial de Origanum vulgare L. e do carvacrol no crescimento de bactérias patogênicas da orofaringe
}

\author{
Effect of Origanum vulgare L. essential oil and carvacrol on the growth of pathogenic bacteria in \\ the oropharynx \\ Efecto del aceite esencial de Origanum vulgare L. y carvacrol sobre el crecimiento de bacterias \\ patógenas en la orofaringe
}

Recebido: 14/01/2021 | Revisado: 17/01/2021 | Aceito: 19/01/2021 | Publicado: 23/01/2021

Letícia Lopes Leuthier
ORCID: https://orcid.org/0000-0003-0354-3433
Universidade Federal da Paraíba, Brasil
E-mail: leticiallopes99@ gmail.com
Ellen Caroline Araújo da Silva
ORCID: https://orcid.org/0000-0002-2131-6317
Universidade Federal da Paraíba, Brasil
E-mail: ecaroline710@ gmail.com
Alexandre Almeida Júnior
ORCID: https://orcid.org/0000-0002-9205-5230
Universidade Federal da Paraíba, Brasil
E-mail: alexandre_junior.02@ @otmail.com
Jocianelle Maria Felix Fernandes Nunes
ORCID: https://orcid.org/0000-0002-0449-5085
Universidade Federal da Paraíba, Brasil
E-mail: jocianelle@ gmail.com
Fábio Correia Sampaio
ORCID: https://orcid.org/0000-0003-2870-5742
Universidade Federal da Paraíba, Brasil
E-mail: fcsampa @gmail.com
Isabela Albuquerque Passos Farias
ORCID: https://orcid.org/0000-0002-3601-1698
Universidade Federal da Paraíba, Brasil
E-mail: isabelaapassos@yahoo.com.br

\section{Resumo}

O objetivo do estudo foi avaliar o efeito dos óleos essenciais de Origanum vulgare L. (OEO) e do carvacrol (CAR) no crescimento de bactérias da orofaringe. Para tanto, foram aplicadas as seguintes técnicas microbiológicas: difusão em ágar, concentração inibitória mínima (CIM) e concentração bactericida mínima (CBM). Na análise estatística, foi aplicado o teste ANOVA, com pós teste de Tukey. O nível de significância adotado foi de 5\%. As cepas testadas foram Pseudomonas aeruginosa ATCC 27853, Staphylococcus aureus ATCC 15656, Escherichia coli ATCC 25922 e Klebsiella pneumoniae INCQS 00147. O OEO apresentou atividade antimicrobiana, variando o tamanho dos halos de 21,00 $( \pm 0,20) \mathrm{mm}$, para E. coli, a 33,56 $( \pm 14,32) \mathrm{mm}$, para $S$. aureus. No teste de CIM, o CAR obteve menor concentração para a $K$. pneumoniae e maior concentração para $S$. aureus. A CBM mostrou valores semelhantes a CIM. O óleo essencial de Origanum vulgare L. e o carvacrol apresentaram efeito inibitório no crescimento de bactérias da orofaringe.

Palavras-chave: Origanum; Testes de sensibilidade a antimicrobianos por disco-difusão; Viabilidade microbiana.

\begin{abstract}
The aim of the study was to evaluate the effect of the essential oils of Origanum vulgare L. (EOO) and carvacrol (CAR) without growth from bacteria oropharynx. The microbiological techniques were applied: agar diffusion method, minimum inhibitory concentration (MIC) and minimum bactericidal concentration (MBC). The ANOVA test was applied, with the Tukey test. The level of significance adopted was 5\%. Tested strains were Pseudomonas aeruginosa ATCC 27853, Staphylococcus aureus ATCC 15656, Escherichia coli ATCC 25922 and Klebsiella pneumoniae INCQS 00147. The EOO showed antimicrobial activity, varying the size of the halos from 21.00 ( \pm $0.20) \mathrm{mm}$, for $E$. coli, to $33.56( \pm 14.32) \mathrm{mm}$, for $S$. aureus. The CAR has MIC lower for K. pneumoniae and a higher concentration for $S$. aureus. The MBC showed similar values the MIC. Origanum vulgare L. essential oil and carvacrol showed an inhibitory effect on the growth of bacteria in the oropharynx.
\end{abstract}

Keywords: Origanum; Disk Diffusion Antimicrobial Tests; Microbial viability. 


\begin{abstract}
Resumen
El objetivo del estudio fue evaluar el efecto de los aceites esenciales de Origanum vulgare L. (AEO) y carvacrol (CAR) sobre el crecimiento de bacterias en la orofaringe. Para ello se aplicaron las siguientes técnicas microbiológicas: difusión en agar, concentración mínima inhibitoria (CMI) y concentración mínima bactericida (CMB). En el análisis estadístico se aplicó la prueba ANOVA, con postest de Tukey. El nivel de significancia adoptado fue del 5\%. Las cepas ensayadas fueron Pseudomonas aeruginosa ATCC 27853, Staphylococcus aureus ATCC 15656, Escherichia coli ATCC 25922 y Klebsiella pneumoniae INCQS 00147. La AEO mostró actividad antimicrobiana, variando el tamaño de los halos desde $21,00( \pm 0,20) \mathrm{mm}$, hasta $E$. coli, a 33,56 $( \pm 14,32) \mathrm{mm}$, para $S$. aureus. En la prueba de CMI, el CAR obtuvo una menor concentración para $K$. pneumoniae y una mayor concentración para S. aureus. CMI mostró valores similares a CMB. El aceite esencial de Origanum vulgare L. y carvacrol tuvo un efecto inhibidor sobre el crecimiento de bacterias en la orofaringe.
\end{abstract}

Palabras clave: Origanum; Pruebas Antimicrobianas de difusión por disco; Viabilidad microbiana.

\title{
1. Introdução
}

A microbiota colonizadora e oportunista da cavidade bucal e orofaringe representam papel importante no processo de desenvolvimento das infecções nosocromiais, associada a ventilação mecânica em pacientes críticos, uma vez que o biofilme dental e lingual pode ser reservatório desses micro-organismos. Assim, algumas estratégias para prevenir a colonização da orofaringe e do trato gastrintestinal têm sido investigadas, como o uso de solução antisséptica para manutenção da higiene bucal regular (Freitas \& Cruz, 2020; Vasconcelos et al., 2019).

Dentre as matérias-primas de soluções antissépticas, destacam-se os óleos essenciais com propriedades terapêuticas. Eles são amplamente utilizados nas indústrias farmacêutica, alimentícia, de perfumaria e, mais recentemente, na medicina (Cabrera-Contreras et al., 2020).

Os óleos essenciais são reconhecidos como seguros pela Administração de Alimentos e Medicamentos dos Estados Unidos, e têm sido usados como aromatizantes e conservantes na alimentação humana há centenas de anos (Rattanachaikunsopon \& Phumkhachorn, 2009; Tavares et al., 2015; Wang et al., 2016). Para tal uso, são comumente diluídos em solventes para spray ou são aquecidos para o vapor eliminar odores e inibir o crescimento microbiano (Wang et al., 2016).

Segundo Khan et al. (2018), o Origanum vulgare L. é uma planta perene de tamanho médio, erva aromática do gênero Origanum. Essa espécie é considerada uma das plantas aromáticas mais amplamente utilizadas. Seus óleos voláteis mono e sesquiterpenóides que constituem até $70 \%$ da totalidade do óleo, podem variar sua constituição a depender da área geográfica e da forma do cultivo da planta.

$\mathrm{Na}$ inibição do crescimento microbiano, o óleo essencial de orégano (OEO) apresentou atividade de inibição do crescimento de Klebsiella pneumoniae (Vasconcelos et al., 2019), Streptococcus resistente a Eritromicina (Magi, Marini \& Facinelli, 2015), Streptococcus pyogenes (Wijesundara \& Rupasinghe, 2018) e Pseudomonas syringae, além de reduzir produção de fator de virulência dessa bactéria (Carezzano et al., 2017). A baixa concentração do OEO foi suficiente para prevenir crescimento de várias bactérias presentes em infecções, com efeito similar ao antimicrobiano Ampicilina (Özkalp et al., 2010). Acredita-se na sua atividade sobre bactérias de infecções respiratórias com efeito na prevenção e erradicação de biofilme (Wijesundara \& Rupasinghe, 2018).

O monoterpeno fenólico carvacrol é o fitoconstituinte majoritário do óleo essencial de Origanum vulgare L., representando 91,6\% dos constituintes, com ação antibacteriana comprovada para Streptococcus resistente a Eritromicina (Magi, Marini \& Facinelli, 2015).

Considerando que a atividade antimicrobiana de diversos óleos essenciais tem sido discutida como promissora no combate a diversos patógenos (Tardugno et al., 2017), os objetivos desse estudo são avaliar o efeito do óleo essencial de Origanum vulgare L. e do fitoconstituinte carvacrol no crescimento de bactérias da orofaringe. A hipótese nula do presente estudo foi: não há efeito do óleo essencial de Origanum vulgare L. e do carvacrol no crescimento de bactérias da orofaringe. 


\section{Metodologia}

\subsection{Componentes a serem testados}

O óleo essencial de Origanum vulgare L. foi obtido na empresa Laslo Indústria e Comércio Ltda (São Paulo, Brasil) e o Digluconato de Clorexidina a 20\%, da empresa Sigma-Aldrich® (São Paulo, Brasil). Os produtos foram solubilizados em dimetilsufóxido (DMSO) a 10\% e TWEEN 80 a $3 \%$ (Diadema, SP, Brazil) na proporção 1:1 de agentes solubilizantes. DMSO foi utilizado como controle negativo e Digluconato de Clorexidina foi o controle positivo em todos os testes antibacterianos do presente estudo.

O óleo essencial de Origanum vulgare L. (OEO) tem origem da Turquia, de agricultura convencional, extraído da folha pela técnica à vapor. A composição cromatográfica de OEO é de 76,5\% e 4,3\% de carvacrol e timol, respectivamente (Grupo Laszlo@, Minas Gerais, Brasil).

\subsection{Cepas bacterianas}

Para o presente estudo foram utilizadas cepas da American Type Culture Collection (ATCC), Instituto Nacional de Controle de Qualidade em Saúde (INCQS): Pseudomonas aeruginosa ATCC 27853, Staphylococcus aureus ATCC 15656, Escherichia coli ATCC 25922 e Klebsiella pneumoniae INCQS 00147. As cepas estavam armazenadas em caldo Infusão Cérebro e Coração (BHI) acrescido de $15 \%$ de glicerol à temperatura de $-70{ }^{\circ} \mathrm{C}$.

\subsection{Difusão em ágar}

O método de difusão em ágar foi conduzido com o óleo essencial e carvacrol, e as cepas bacterianas padronizadas na escala 0,5 de Mc Farland. Orifícios de $8 \mathrm{~mm}$ foram realizados em placas de Petri contendo meio de cultura BHI ágar. As placas foram mantidas por 30 minutos a temperatura ambiente para pré-difusão antes da incubação a $37^{\circ} \mathrm{C}$ em microaerofilia, de acordo com a cepa, para mensuração do halo de inibição com 24 horas (adaptado de NCCLS, 2003a). Gluconato de clorexidina $0,12 \%$ foi o controle positivo. $\mathrm{O}$ teste foi realizado em triplicata.

\subsection{Concentração inibitória mínima}

A concentração inibitória mínima (CIM) da solução foi determinada pelo método de microdiluição em placa de 96 poços de acordo com Committee for Clinical laboratory Standard (Cockerill, 2012; NCCLS, 2003b). Duas filas de poços foram preparadas iniciando com óleo e fitoconstituintes na maior concentração. Cada poço apresentou caldo do meio de cultura BHI, substâncias testadas e inóculo de patógenos da orofaringe (20\%) em concentrações decrescentes das substâncias. A placa foi incubada em estufa microbiológica a $37^{\circ} \mathrm{C}$ por $24-48 \mathrm{~h}$. Após esse período, $35 \mu \mathrm{L}$ do indicador de oxidaçãoredução resazurina 0,01\% (Sigma-Aldrich ${ }^{\circledR}$, São Paulo, Brasil) foi dispensado em cada poço. Os poços que mudaram a cor de azul para róseo após 40 minutos de contato com corante, apresentam crescimento microbiano. O valor da CIM foi definido no poço de menor concentração da substância testada que permaneceu azul. O teste foi realizado em triplicata.

\subsection{Concentração bactericida mínima}

A concentração bactericida mínima (CBM) foi verificada pelo cultivo de $30 \mu \mathrm{L}$ da solução dos poços que permaneceram azuis no teste de CIM. Três gotas foram dispensadas em placas de petri contendo meio BHI ágar. A CBM foi a menor concentração capaz de inibir completamente o crescimento bacteriano. $\mathrm{O}$ teste foi realizado em triplicata.

\subsection{Análise estatística}

Os dados foram conduzidos para banco de dados informatizados, e analisados pela estatística descritiva (média, 
desvio-padrão, mediana). Foram aplicados os testes ANOVA e Tukey para comparar médias do teste de halo de difusão. O nível de significância adotado foi de 5\%.

\section{Resultados e Discussão}

O presente estudo abordou a atividade antimicrobiana do óleo essencial de orégano e carvacrol como uma alternativa para ingredientes ativos em insumos de higiene oral contra bactérias da orofaringe.

O OEO apresentou atividade antimicrobiana pelo teste de difusão, variando o tamanho dos halos de $21,00( \pm 0,20)$ a 33,56 ( $\pm 14,32) \mathrm{mm}$, para E. coli e $S$. aureus, respectivamente. Houve diferença estatisticamente significativa entre OEO e CHX apenas para P. aeruginosa (Tabela 1). Atividade antimicrobiana em placa de petri para S. aureus e E. coli também foi observado em estudo anterior, mas os autores não realizaram medição do halo de inibição, apenas presença ou ausência de crescimento de colônias bacterianas (Cabrera-Contreras et al., 2020).

De forma semelhante, o carvacrol manifestou ação eficaz com halo de inibição entre $26,93 \pm 1,95 \mathrm{~mm}$, para $K$. pneumoniae, e 35,96 \pm 7,23 mm, para E. coli, com diferença estatisticamente em relação ao controle positivo para esta última cepa (Tabela 1). Valores semelhantes de halo de inibição para K. pneumoniae e E. coli foram observados em outros estudos (Vasconcelos et al., 2019; Santos et al., 2020). Enquanto para S. aureus, o halo de inibição do OEO foi duas vezes maior que o observado por Santos et al. (2020).

Tabela 1 - Distribuição dos halos de inibição bacteriana dos óleos e fitoconstituintes de acordo com as cepas de bactérias da orofaringe.

\begin{tabular}{cccc}
\hline & & Halo de inibição $(\mathrm{mm})$ & \\
Cepa bacteriana & OEO & CAR & CHX \\
& Média $( \pm \mathrm{DP})$ & Média $( \pm \mathrm{DP})$ & Média $( \pm \mathrm{DP})$ \\
\hline S. aureus & $33,56^{\mathrm{a}}( \pm 14,32)$ & $28,06^{\mathrm{a}}( \pm 1,65)$ & $20,53^{\mathrm{a}}( \pm 6,49)$ \\
P. aeruginosa & $28,73^{\mathrm{a}}( \pm 1,07)$ & $34,73^{\mathrm{b}, \mathrm{c}}( \pm 1,85)$ & $12,13^{\mathrm{c}}( \pm 1,34)$ \\
K. pneumoniae & $24,06^{\mathrm{a}}( \pm 4,63)$ & $26,93^{\mathrm{a}}( \pm 1,95)$ & $26,93^{\mathrm{a}}( \pm 9,05)$ \\
E. coli & $21,00^{\mathrm{a}}( \pm 0,20)$ & $35,96^{\mathrm{b}}( \pm 7,23)$ & $17,30^{\mathrm{a}, \mathrm{c}}( \pm 1,12)$ \\
\hline
\end{tabular}

Fonte: Autores (2021).

OEO - óleo essencial de orégano; CAR - Carvacrol; CHX - Digluconato de Clorexidina.

Letras diferentes na linha indicam diferença estatisticamente significativa $(\mathrm{p}<0,05)$. Teste ANOVA, teste de Tukey.

Os valores de CIM de OEO e CAR foram semelhantes para S. aureus (Tabela 2). A atividade antimicrobiana para $S$. aureus e as demais bactérias do presente estudo foi testada na fase de crescimento (log) bacteriano. Boa atividade do OEO também foi verificada previamente na fase estacionária para S. aureus (Xião et al., 2000).

O OEO testado no presente estudo é de origem da Turquia com quantidade de carvacrol semelhante ao OEO da Arábia Saudita, que apresentou atividade antimicrobiana significativa contra S. aureus, P. aeruginosa e E. coli (Khan et al., 2018). Para K. Pneumoniae, o OEO apresentou boa atividade, corroborando achados de OEO originário da Moldávia, com $72 \%$ de carvarol (Vasconcelos et al., 2019). Os resultados das Tabelas 2 e 3 reforçam o potencial antibacteriano do carvacrol contra K. pneumoniae isolado ou combinado com outros antimicrobianos como verificado no estudo de Cordeiro et al. (2020). 
Tabela 2 - Valores de Concentração inibitória mínima dos óleos e fitoconstituintes de acordo com as cepas de bactérias da orofaringe.

\begin{tabular}{cccc}
\hline & \multicolumn{3}{c}{ Concentração inibitória mínima $(\mu \mathrm{g} / \mathrm{mL})$} \\
Cepa bacteriana & OEO & CAR & CHX \\
\hline S. aureus & 5000,00 & 5000,00 & 1458,33 \\
P. aeruginosa & 4166,67 & 4166,67 & 2500,00 \\
K. pneumoniae & 5000,00 & 3333,33 & 2916,67 \\
E. coli & 5000,00 & 4166,67 & 3750,00 \\
\hline
\end{tabular}

Fonte: Autores (2021).

OEO - Óleo essencial de orégano; CAR - Carvacrol; CHX - Digluconato de Clorexidina

A Concentração Bactericida Mínima (CBM) mostrou valores semelhantes entre as cepas para OEO, bem como, foi similar a CIM, apresentando pequenas variações entre $3333,33 \mu \mathrm{g} / \mathrm{mL}$ a $5000 \mu \mathrm{g} / \mathrm{mL}$ (Tabela 3). O CAR revelou menor concentração bactericida frente a $P$. aeruginosa.

Tabela 3 - Valores de Concentração bactericida mínima dos óleos e fitoconstituintes de acordo com as cepas de bactérias da orofaringe.

\begin{tabular}{cccc}
\hline & & Concentração bactericida mínima $(\mu \mathrm{g} / \mathrm{mL})$ & CHX \\
Cepa bacteriana & OEO & CAR & 4166,67 \\
S. aureus & 5000 & 5000 & 4166,67 \\
P. aeruginosa & 5000 & 4166,67 & 3333,33 \\
K. pneumoniae & 5000 & 5000 & 5000 \\
E. coli & 5000 & 5000 & \\
\hline
\end{tabular}

Fonte: Autores (2021).

OEO - Óleo essencial de orégano; CAR - Carvacrol; CHX - Digluconato de Clorexidina

\section{Conclusão}

O óleo essencial de Origanum vulgare L. e o carvacrol apresentaram efeito inibitório no crescimento de bactérias da orofaringe.

Pesquisas futuras na temática podem ser dedicadas ao desenvolvimento e avaliação de insumos de higiene bucal contendo óleo essencial de orégano e/ou carvacrol.

\section{Agradecimentos}

Ao CNPq (Conselho Nacional de Desenvolvimento Científico e Tecnológico) pelo auxílio financeiro na forma de bolsa de estudos. 


\section{Referências}

Cabrera-Contreras, R., Morelos-Ramírez, R., Quiróz-Ríos, J. P., \& Muñoz-Quiróz, D. (2020). Antibacterial Effects of Oregano Essential Oil (OEO) and Its Potential Applications. Microbiology Research Journal International, 30 (4), 19-25.

Carezzano, M. E., Sotelo, J. P., Primo, E., Reinoso, E. B., Paletti Rovey, M. F., Demo, M. S., Giordano, W.F. \& Oliva, de las M. (2017). Inhibitory effect of Thymus vulgaris and Origanum vulgare essential oils on virulence factors of phytopathogenic Pseudomonas syringae strains. Plant Biology, 19 (4), $599-607$.

CLSI. (2015). Methods for Dilution Antimicrobial Susceptibility Tests for Bacteria That Grow Aerobically: Approved Standard. Clinical and Laboratory Standards Institute, 35(2).

Cordeiro, L. V., Figueiredo, P. T. R., Sousa, A. P., Andrade Jr, F. P., Souza, H. D. S., Araújo, D. L., Sobreira, A. L. C. \& Lima, E. O. (2020). Association of carvacrol with ceftazidime and cefepime against Klebsiella pneumoniae. Research, Society and Development, 9 (7), e264974089.

Cui, H., Zhang, C., Li, C., \& Lin, L. (2019). Antibacterial mechanism of orégano essential oil. Industrial Crops \& Products, 139, 1-9.

Freitas, L. M. A. \& Cruz, I.C.F. (2020). Nursing evidence-based practice guidelines for self-care: oral hygiene in ICU - Systematic Literature Review. Journal of Specialized Nursing Care, 12(1).

Kachur, K. \& Suntres, Z. (2019). The antibacterial properties of phenolic isomers, carvacrol and thymol. Critical Reviews in Food Science and Nutrition, 60 (18), 3042-3053.

Khan, M., Khan, S. T., Khan, N. A., Mahmood, A., Al-Kedhairy, A. A., \& Alkhathlan, H. Z. (2018). The composition of the essential oil and aqueous distillate of Origanum vulgare L. growing in Saudi Arabia and evaluation of their antibacterial activity. Arabian Journal of Chemistry,11(8), 1189-1200.

Magi, G., Marini, E. \& Facinelli, B. (2015). Antimicrobial activity of essential oils and carvacrol, and synergy of carvacrol and erythromycin, against clinical, erythromycin-resistant Group A Streptococci. Frontiers in Microbiology, 6(165), 1-7.

NCCLS. (2003a). Performance Standards for Antimicrobial Disk Susceptibility Tests; Approved Standard- Eighth Edition. Clinical and Laboratory Standards Institute. M2-A8.

NCCLS. (2003b). Methods for Dilution Antimicrobial Susceptibility Tests for Bacteria That Grow Aerobically; Approved Standard-Sixth Edition. Clinical and Laboratory Standards Institute. M7-A6.

Özalp. B., Sebgi, F. \& Özcan, M. \& Özcan, M. M. (2010). The antibacterial activity of essential oil of oregano (Origanum vulgare L.). Journal of Food, Agriculture and Environment, 8(2), 272-274.

Rattanachaikunsopon, P. \& Phumkahchorn, P. (2009). Shallot (Allium ascalonicum L.) oil: Diallyl sulfide content and antimicrobial activity against foodborne pathogenic bacteria. African Journal of Microbiology Research, 3(11), 747-750.

Santos, J. R. N. dos, Teles A. M., Ferreira, C. G. \& Mouchrek, A. N. (2020). Evaluation of the bactericidal and antioxidant activity of essential oil and hydroalcoholic extract of oregano (Origanum vulgare). Research, Society and Development, 9 (10), e7829108410.

Tardugno, R., Pellati, F., Iseppi, R., Bondi, M., Bruzzesi, G., \& Benvenuti, S. (2017). Phytochemical composition and in vitro screening of the antimicrobial activity of essential oils on oral pathogenic bacteria. Natural Product Research, 32(5), 544-551.

Tavares, A. G., Monte, D. F. M. D., Albuquerque, A. D. R., Sampaio, F. C., Magnani, M., Siqueira Júnior, J. P. D., \& Souza, E. L. D. (2015). Habituation of enterotoxigenic Staphylococcus aureus to Origanum vulgare L. essential oil does not induce direct-tolerance and cross-tolerance to salts and organic acids. Brazilian Journal of Microbiology, 46(3), 835-840.

Vasconcelos, N. G., Croda, J., Silva, K. E., Motta, M. L. L., Maciel, W. G., Limiere, L. C., \& Simionatto, S. (2019). Origanum vulgare L. essential oil inhibits the growth of carbapenem-resistant gram-negative bacteria. Revista da Sociedade Brasileira de Medicina Tropical, $52, \mathrm{e} 2018050$.

Wijesundara, N. M. \& Rupasinghe. H. P. V. (2018). Essential oils from Origanum vulgare and Salvia officinalis exhibit antibacterial and anti-biofilm activities against Streptococcus pyogenes. Microbial Pathogenesis, 117, 118-127.

Xiao, S., Cui, P., Shi, W., \& Zhang, Y. (2020). Identification of essential oils with activity against stationary phase Staphylococcus aureus. BMC complementary medicine and therapies, 20(1), 1-10. 